\title{
Anti-diarrhea effects of polysaccharides from Zingiber officinale rhizome on a rat diarrhea model
}

\author{
Dong-Mei Su, Jun-Biao Long, Xin-Ping Liu, Shu-Bin Li* \\ The Department of Gastroenterology, South of Guang'anmen Hospital, China Academy of Chinese Medical Sciences, Beijing \\ 102618, PR China
}

*For correspondence: Email: lisbcacms@sina.com

\begin{abstract}
Purpose: To investigate the anti-diarrhea effects of polysaccharides extracted from the rhizome of Zingiber officinale (ZRP) on diarrhea induced by folium sennae in rats.

Methods: Response surface methodology (RSM) based on the Box-Benhnken design (BBD) was performed to optimize ZRP extraction. Thereafter, experimental diarrheal rats induced by treatment with folium sennae were used to investigate the anti-diarrhea effects of ZRP. Diarrhea index, dilute stool rate, gastric residual rate, and intestinal propulsive rate were investigated. In addition, the levels of brain-gut peptides including cholecystokinin (CCK), ghrelin, and vasoactive intestinal peptide (VIP) in the small intestine of rats with diarrhea were determined using commercial ELISA kits.

Results: Optimal ZRP extraction was obtained with an extraction time of $1.4 \mathrm{~h}$, ratio of water to the raw material of $30 \mathrm{~mL} / \mathrm{g}$, and extracting 2 times. ZRP treatment at doses of 50, 100, and $200 \mathrm{mg} / \mathrm{kg}$ significantly decreased the dilute stool rate and diarrhea index $(p<0.05)$ and increased gastric residual rate $(p<0.01)$ dose-dependently. ZRP lowered intestinal propulsive rate $(100$ and $200 \mathrm{mg} / \mathrm{kg}, p<0.01)$. All ZRP doses (50, 100 and $200 \mathrm{mg} / \mathrm{kg})$ also significantly reduced the levels of CCK $(p<0.01)$ but increased the levels of ghrelin and VIP $(p<0.01)$ in the small intestine.

Conclusion: ZRP exerts significant anti-diarrhea effects on experimental diarrheal rats induced by folium sennae via regulating the levels of brain-gut peptides. Further studies are required to determine if these effects can also be replicated in humans
\end{abstract}

Keywords: Zingiber officinale, Polysaccharide, Response surface methodology, Anti-diarrhea, Cholecystokinin, Ghrelin, and Vasoactive intestinal peptide

\begin{abstract}
This is an Open Access article that uses a funding model which does not charge readers or their institutions for access and distributed under the terms of the Creative Commons Attribution License (http://creativecommons.org/licenses/by/4.0) and the Budapest Open Access Initiative (http://www.budapestopenaccessinitiative.org/read), which permit unrestricted use, distribution, and reproduction in any medium, provided the original work is properly credited.
\end{abstract}

Tropical Journal of Pharmaceutical Research is indexed by Science Citation Index (SciSearch), Scopus, International Pharmaceutical Abstract, Chemical Abstracts, Embase, Index Copernicus, EBSCO, African Index Medicus, JournalSeek, Journal Citation Reports/Science Edition, Directory of Open Access Journals (DOAJ), African Journal Online, Bioline International, Open-J-Gate and Pharmacy Abstracts

\section{INTRODUCTION}

Diarrhea, characterized by high-frequency and liquid defecation, is one of the most common symptoms of digestive diseases. Diarrhea can have severe sequelae such as barley syndrome, hemolytic uremic syndrome, and even death. [1]. Causes of diarrhea are complicated and include digestive system diseases such as acute enteritis, inflammatory bowel disease, and irritable bowel syndrome, as well as hyperthyroidism, diabetes, and uremia [1].

Herbal medicine and its extracts/constituents are currently considered helpful for curing human diseases with lower toxicities $[2,3]$. The rhizome 
of Zingiber officinale, a known TCM in China, has been commonly used to treat various gastrointestinal diseases, such as abdominal cold-pain, diarrhea, and cough [4]. Modern investigations have demonstrated that the rhizome of $Z$. officinale contains many different constituents, including gingerol, diarylheptanoids, and polysaccharides [5]. However, recent research regarding the curative effects of the $Z$. officinale rhizome on diarrhea primarily focused on its small molecular constituents, and its macromolecular compounds have rarely been studied. Thus, the present study was designed to examine the anti-diarrhea effect of polysaccharides from the rhizome of $Z$. officinale (ZRP) on an experimental rat model of diarrhea.

\section{EXPERIMENTAL}

\section{Chemical reagents}

Rat cholecystokinin (CCK) and vasoactive intestinal peptide (VIP) were purchased from Guangzhou Bian Jin Biological Technology Co., Ltd (Guangzhou, China). Rat ghrelin was obtained from Nanjing Jin Yi Bai Biological Technology Co., Ltd (Nanjing, China). Reserpine was obtained from Shanghai Yi Yan Biological Technology Co., Ltd (Shanghai, China). All other chemical reagents used in this study were of analytical grade.

\section{Animals}

Healthy male rats (weighing 180-220 g) were purchased from the Laboratory Animal Center of the China Academy of Chinese Medical Sciences (Beijing, China). The animals were maintained under controlled conditions with natural lighting and free access to food and water. The animal protocols used were in accordance with the declaration of Helsinki promulgated in 1964 as amended in 1996 [6], and were approved by the Animal Care and Use Committee of South of Guang'anmen Hospital, China Academy of Chinese Medical Sciences (no. CACMS2017115).

\section{Preparation of ZRP}

The rhizome of $Z$. officinale was processed to powder using pulverized machine and extracted with $95 \%$ ethanol for $12 \mathrm{~h}$. After filtration, the residue was air dried and extracted with distilled water by reflux. The extract was left to cool at room temperature and filtered. Subsequently, ethanol was added slowly to a final concentration of $80 \%$. After precipitation overnight at $4^{\circ} \mathrm{C}$, the precipitates were collected by filtering and washed thrice with pure ethanol and acetone, then dried at $50^{\circ} \mathrm{C}$ to obtain the crude polysaccharides hereafter referred to as ZRP. The contents of the polysaccharides were measured by the phenol-sulfuric method as previously reported [7]. Glucose was used as a standard, and the yield (\%) of polysaccharides was calculated as follows:

Yield $(\%)=W_{1} / W_{0} \ldots \ldots \ldots \ldots(1)$

where $W_{1}$ was the content of crude polysaccharides and $W_{0}$ was the weight of dried $Z$. officinale rhizome used.

\section{Experimental design and statistical analysis}

According to our initial extraction results, three major influence factors were identified including extraction time $(A)$, ratio of water to raw material (B), and number of times extracted (C). To obtain an optimal extraction of ZRP, we carried out response surface methodology (RSM) based on Box-Benhnken Design (BBD). The parameters tested were $A: 1,1.5$, and $2 \mathrm{~h} ; \mathrm{B}: 20,30$, and 40 $\mathrm{mL} / \mathrm{g}$; and $\mathrm{C}: 1,2$, and 3 extractions, and for each factor the low, medium, and high levels were coded $-1,0$, and +1 , respectively. Seventeen experiments based on BBD with three center points were performed in random order (Table 1).

All experiments were repeated three times, and the analysis of variance (ANOVA) was carried out to analyze the BBD results using Design Expert software (version 8.0.6, Stat-Ease, Inc., Minneapolis, MN, USA). In addition, response surfaces were drawn to analyze the individual and interactive effects of each test variable on response. Additional confirmatory experiments were performed to verify the validity of the statistical experimental design.

\section{Animal model and groups}

An animal model of diarrhea resulting from spleen yang deficiency induced by folium sennae was established according to previous methods [8]. A total of 50 male rats were divided into 5 groups $(n=10)$, which consisted of the normal group, model group, and 3 ZRP-treated model groups $(50,100$, and $200 \mathrm{mg} / \mathrm{kg})$. Rats in the model and ZRP treatment groups were administered $4^{\circ} \mathrm{C}$ water extract of folium sennae $(10 \mathrm{~g} / \mathrm{kg})$ once a day by gavage, then were injected with reserpine $(0.5 \mathrm{mg} / \mathrm{kg})$ subcutaneously and swam in $30^{\circ} \mathrm{C}$ water on alternating days. 
Table 1: Box-behnken experimental design with independent variables

\begin{tabular}{lcccc}
\hline Run & A & B & C & Yield (\%) \\
\hline 1 & 0.00 & 1.00 & -1.00 & 11.18 \\
2 & 0.00 & 0.00 & 0.00 & 12.22 \\
3 & 0.00 & -1.00 & 1.00 & 11.06 \\
4 & -1.00 & -1.00 & 0.00 & 11.51 \\
5 & 1.00 & 0.00 & 1.00 & 10.46 \\
6 & 0.00 & -1.00 & -1.00 & 11.43 \\
7 & 0.00 & 0.00 & 0.00 & 12.29 \\
8 & 1.00 & 0.00 & -1.00 & 11.53 \\
9 & 0.00 & 0.00 & 0.00 & 12.37 \\
10 & -1.00 & 0.00 & -1.00 & 11.48 \\
11 & 1.00 & -1.00 & 0.00 & 11.29 \\
12 & 1.00 & 1.00 & 0.00 & 11.17 \\
13 & 0.00 & 0.00 & 0.00 & 12.28 \\
14 & 0.00 & 0.00 & 12.11 \\
15 & 0.00 & 1.00 & 0.00 & 11.46 \\
16 & -1.00 & 1.00 & 1.00 & 10.59 \\
\hline
\end{tabular}

These treatments were continued for 3 weeks. Normal rats were given distilled water by gavage in place of folium sennae and injected with an equal volume of normal saline rather than reserpine. After 3 weeks, the $3 \mathrm{ZRP}$ treatment groups were given ZRP solutions $\left(38^{\circ} \mathrm{C}\right)$ at doses of 50,100 , and $200 \mathrm{mg} / \mathrm{kg}$ by gavage, and normal and model rats were treated with distilled water.

\section{Effect of ZRP on dilute stool rate and diarrhea index of rats}

After the last administration of ZRP, rats in each group were fasted for $12 \mathrm{~h}$, but allowed access to water. Each rat was placed in a squirrel cage with filter paper for $5 \mathrm{~h}$ of observation and recording of the defecation of each rat. Dilute stool rate (DSR) and diarrhea index (DI) were used to evaluate the effects of ZRP on folium sennae-induced diarrhea in rats and were calculated as in Eqs 2 and 3, respectively.

$\operatorname{DSR}(\%)=\left(N_{1} / N_{2}\right) 100$

where $N_{1}$ was the number of dilute feces and $N_{2}$ was the total feces number of the rat.

$\mathrm{DI}=\mathrm{DSR}-\mathrm{DD}$

where $\mathrm{DD}=$ degree of diarrhea. Dilute stool was defined as the presence of stains on the filter paper around the stool. The degree of diarrhea was determined by measuring the stain diameter with a ruler and classified as follows: $1,<1 \mathrm{~cm} ; 2$, $1-2 \mathrm{~cm} ; 3,2.1-3 \mathrm{~cm} ; 4,3.1-4 \mathrm{~cm}$.

Effect of ZRP on gastric residual rate and intestinal propulsive rate of rats

After the last administration of ZRP and 12-h fast, rats in each group were fed a $10 \%$ charcoal powder solution (dissolved in $2 \% \mathrm{CMC}-\mathrm{Na}$ ) at a dose of $20 \mathrm{~mL} / \mathrm{kg}$. After $20 \mathrm{~min}$, the rats were sacrificed and the stomach and small intestine were removed. The total weight of the stomach was measured. After washing out the contents of the stomach, the net weight of the stomach was also measured. The full length of the small intestine and propulsion length of the charcoal powder were measured. Gastric emptying rate $(G)$ and intestinal propulsive rate $(P)$ of rats were determined as in Eqs 4 and 5, respectively.

Gastric residual rate $(\%)=\left\{\left(W_{1}-W_{2}\right) / W_{1}\right\} 100 \ldots$

where $W_{1}$ and $W_{2}$ are the total and net weights of the stomach.

$P(\%)=\left(L_{1} / L_{2}\right) 100$

where $L_{1}$ is the full length of the small intestine and $L_{2}$ is the propulsion length of charcoal powder in the small intestine.

Effects of ZRP on CCK, ghrelin, and VIP levels in the small intestine of rats

The levels of CCK, ghrelin, and VIP proteins in the small intestine of rats were determined using commercial ELISA kits according to the manufacturer's instructions.

\section{RESULTS}

\section{Response surface analysis}

The predicted response $Y$ (yield of ZRP) was obtained by the following second-order polynomial equation: $\mathrm{Y}=12.25-0.16 \mathrm{~A}-0.11 \mathrm{~B}$ $-0.28 C-0.018 A B-0.21 A C-0.055 B C-0.39$ $A^{2}-0.51 B^{2}-0.68 C^{2}$. ANOVA was used to evaluate the significance of the data and to test 
for adequacy and fitness by ANOVA. The results of the analysis of goodness-of-fit, variance, and adequacy of the mode are presented in Table 2.

The determination coefficient and adjusted determination coefficient were 0.9834 and 0.9620, respectively, which demonstrated that the model was highly significant. It also indicated a high degree of correlation between the observed and predicted values. A low coefficient of variation (2.71) showed a very high degree of precision and reliability of the experimental values. In addition, statistical analysis confirmed that the proposed model was highly significant, possessing a very low $p$ value $(p<0.0001)$. The $F$ value $(2.10)$ and $p$ value $(0.2425)$ of lack of fit indicated that it was not significant relative to the pure error, which showed that the model equation was adequate for predicting ZRP yield under any combination of values of the variables.

Figure 1 presents the 2D contour plots and 3D response surface of the regression equation that revealed the effects of independent variables and their mutual interaction on the extraction yield of ZRP. Importantly, the optimum condition for yield of ZRP was obtained: extraction time of $1.43 \mathrm{~h}$, ratio of water to raw material of $29.02 \mathrm{~mL} / \mathrm{g}$, and 1.82 extractions. The theoretical highest yield of ZRP was predicted to be $12.30 \%$ by the developed model. Three replicate verification experiments were performed at the simplified conditions of extraction time $1.4 \mathrm{~h}$, ratio of water to raw material $30 \mathrm{~mL} / \mathrm{g}$, and 2 extractions. The average yield of ZRP obtained from real experiments was $11.98 \%$, validating the RSM model.
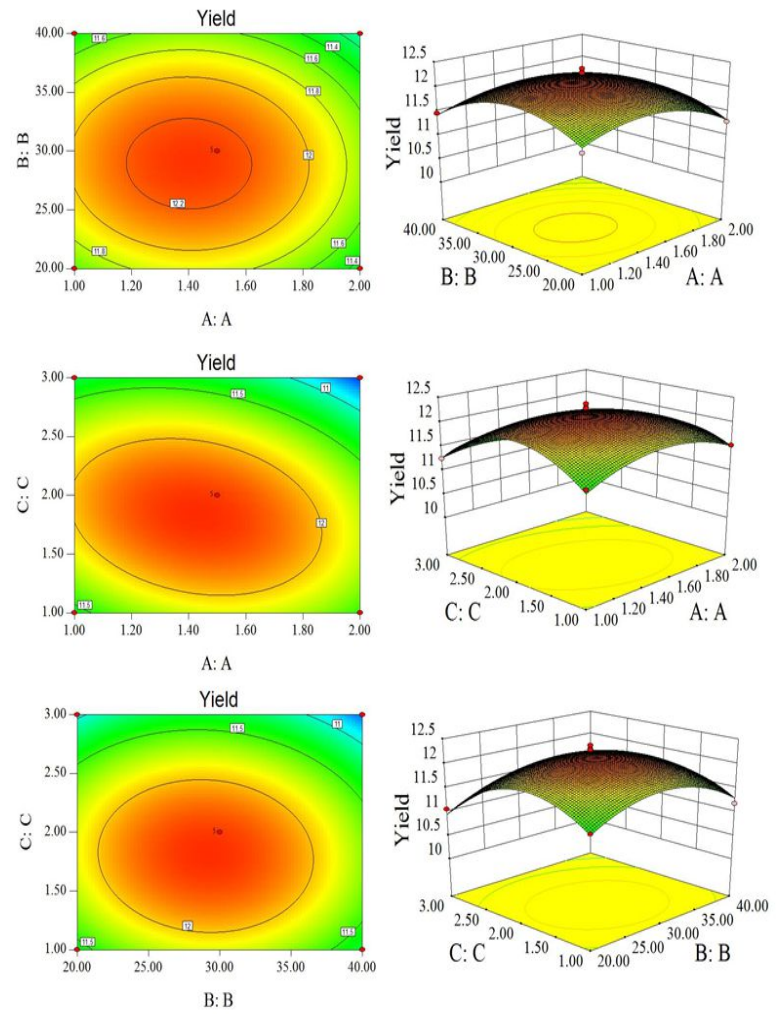

Figure 1: Response surface methodology plots

Effect of ZRP on dilute stool rate and diarrhea index of rats with diarrhea induced by spleen yang deficiency

The effects of ZRP on dilute stool rate and diarrhea index of rats are shown in Figure 2. The dilute stool rate and diarrhea index of rats in 3 ZRP-treated groups $(50,100$, and $200 \mathrm{mg} / \mathrm{kg}$ ) significantly decreased relative to those of model rats in a dose-dependent manner $(p<0.05)$.

Table 2: Analysis of variance of the experimental results of the Box-behnken design

\begin{tabular}{|c|c|c|c|c|c|c|c|}
\hline Source & & Sum of squares & df & Mean Square & & F-value & $P$-value \\
\hline Model & & 5.21 & 9 & 0.58 & & 42.13 & $<0.0001$ \\
\hline A & & 0.20 & 1 & 0.20 & & 14.21 & 0.0070 \\
\hline B & & 0.099 & 1 & 0.099 & & 7.21 & 0.0313 \\
\hline $\mathrm{C}$ & & 0.64 & 1 & 0.64 & & 46.46 & 0.0002 \\
\hline$A B$ & & $1.225 \mathrm{E}-003$ & 1 & $1.225 \mathrm{E}-003$ & & 0.089 & 0.7739 \\
\hline$A C$ & & 0.18 & 1 & 0.18 & & 12.84 & 0.0089 \\
\hline $\mathrm{BC}$ & & 0.012 & 1 & 0.012 & & 0.88 & 0.3793 \\
\hline$A^{2}$ & & 0.64 & 1 & 0.64 & & 46.78 & 0.0002 \\
\hline$B^{2}$ & & 1.08 & 1 & 1.08 & & 78.37 & $<0.0001$ \\
\hline $\mathrm{C}^{2}$ & & 1.97 & 1 & 1.97 & & 143.03 & $<0.0001$ \\
\hline Residual & & 0.096 & 7 & 0.014 & & & \\
\hline Lack of Fit & & 0.059 & 3 & 0.020 & & 2.10 & 0.2425 \\
\hline Pure Error & & 0.037 & 4 & $9.330 \mathrm{E}-003$ & & & \\
\hline Cor Total & & 5.31 & 16 & & & & \\
\hline $\begin{array}{l}\text { Standard } \\
\text { Deviation }\end{array}$ & Mean & C.V.\% & Press & $\mathrm{R}^{2}$ & $\mathrm{R}^{2}$ Adj & $\mathrm{R}^{2}$ Pred & $\begin{array}{l}\text { Adequate } \\
\text { precision }\end{array}$ \\
\hline 0.17 & 6.43 & 2.71 & 2.52 & 0.9834 & 0.9620 & 0.8030 & 19.187 \\
\hline
\end{tabular}




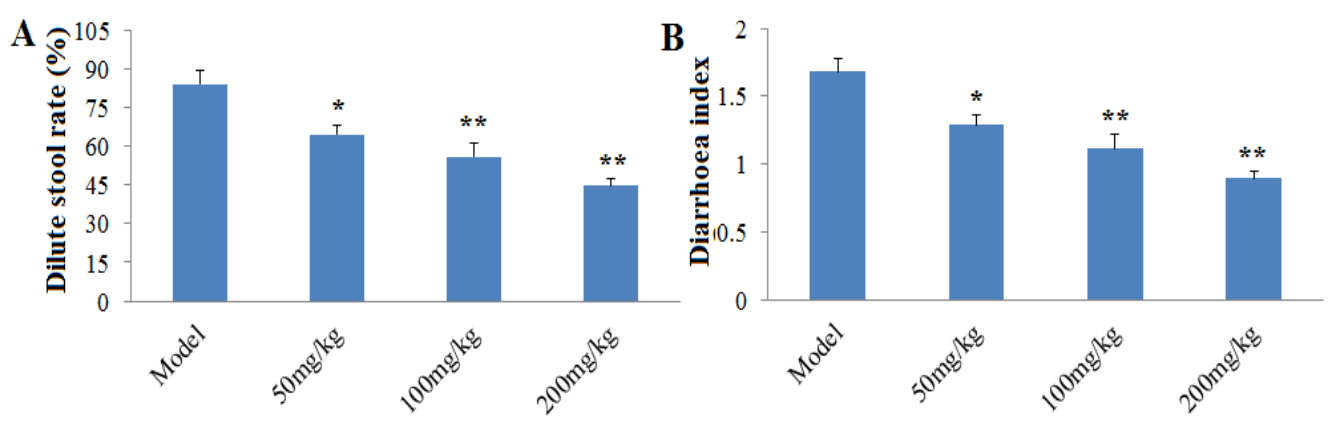

Figure 2: Effect of ZRP on dilute stool rate and diarrhea index of rats with spleen yang deficiency. Data were expressed as mean \pm SD $(n=10)$, and asterisks indicate significant differences; ${ }^{* *} p<0.01$ vs model
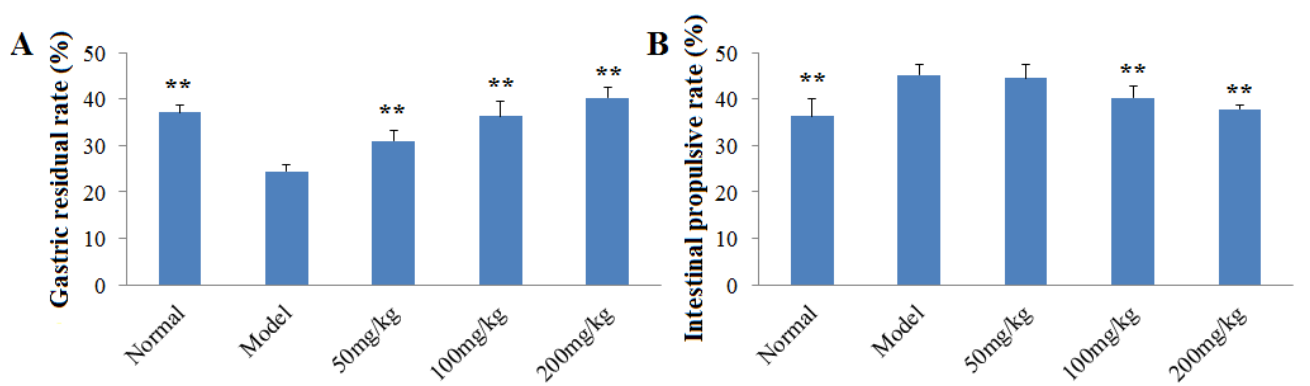

Figure 3: Effects of ZRP on gastric residual rate and intestinal propulsive rate of rats with deficiency of spleen yang. (A) gastric residual rate; (B) intestinal propulsive rate. Data were expressed as mean $\pm \operatorname{SD}(n=10) ;{ }^{* *} p<$ 0.01 vs. model
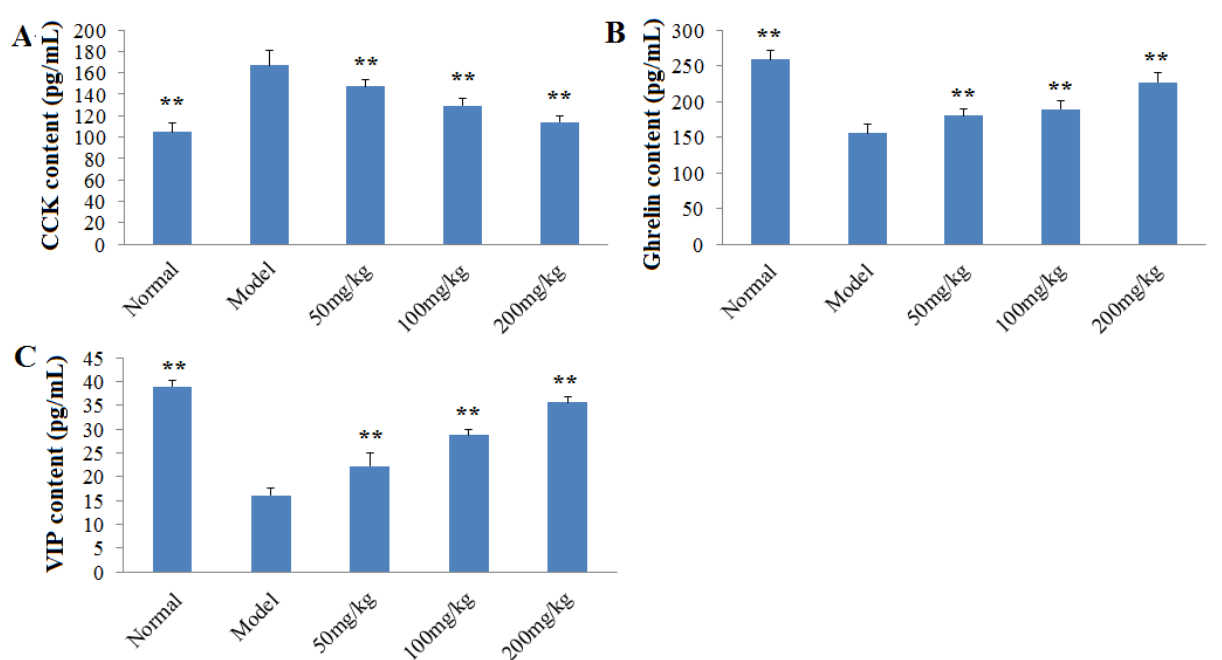

Figure 4: Effects of ZRP on brain-gut peptide levels in the small intestine of rats with deficiency of spleen yang. (A) CCK; (B) Ghrelin; (C) VIP. Data were expressed as mean \pm SD $(n=10)$; ${ }^{* *} p<0.01$ vs. model

\section{Effect of ZRP on gastric residual rate and intestinal propulsive rate of rats}

Figure 3 presents the effect of ZRP on the gastric residual rates and intestinal propulsive rates of rats with diarrhea caused by deficiency of spleen yang. As shown in Figure $3 \mathrm{~A}$, the gastric residual rates of rats in the model group were significantly lower when compared with those of normal rats $(p<0.01)$, and ZRP at doses of 50, 100, and 200 $\mathrm{mg} / \mathrm{kg}$ increased the gastric residual rates of diarrhea model rats dose-dependently $(p<0.01)$.
The intestinal propulsive rates of model rats were significantly higher than those of normal rats $(p<$ 0.01 ), and these rates declined in ZRP-treated groups (100 and $200 \mathrm{mg} / \mathrm{kg}$ ) when compared with model rats $(p<0.01)$ (Figure $3 \mathrm{~B})$.

\section{Effects of ZRP on CCK, ghrelin, and VIP levels in the small intestine of rats}

The effects of ZRP on CCK, ghrelin, and VIP protein content in the small intestine of rats with diarrhea are shown in Figure 4. It can be 
observed that the level of CCK in the small intestine of model rats was significantly increased relative to that of rats in the normal group $(p<0.01)$, and ZRP at doses of 50, 100, and $200 \mathrm{mg} / \mathrm{kg}$ significantly decreased the content of CCK dose-dependently $(p<0.01)$ (Figure 4A). Conversely, ghrelin (Figure 4B) and VIP (Figure 4C) levels in the small intestine of model rats were lower relative to those of normal rats $(p<0.01)$. The ghrelin and VIP content in the small intestine of rats in 3 ZRP-treated groups were significantly increased, dosedependently, relative to model rats $(p<0.01)$.

\section{DISCUSSION}

RSM has been considered an effective statistical method for optimizing complicated processes because it is less laborious and time-consuming than other methods, and it has been widely used to optimize polysaccharide extraction processes $[9,10]$. Furthermore, the BBD is an effective method for investigating the optimization of the extraction process with fewer experiments required [11]. Therefore, in the present study, the process of polysaccharide extraction from the $Z$. officinale rhizome was optimized using RSM based on BBD. This study revealed that the model established by RSM and based on BBD was satisfactory and accurate for optimizing the extraction of ZRP.

An animal diarrhea model induced by folium sennae, which depletes spleen yang, is often used to evaluate the anti-diarrhea effect of medicine [12]. Thus, diarrhea caused by folium sennae was used to evaluate the effect of ZRP on diarrhea in rats. The dilute stool rate and diarrhea index is often used to evaluate the effect of some medicine on diarrhea [13]. In addition, diarrhea can cause certain disorders of the stomach and intestines, and therefore the gastric residual rate and intestinal propulsive rate are also often used as indicators of diarrhea [14]. In the present investigation, the dilute stool rate, diarrhea index, gastric residual rate, and intestinal propulsive rate were analyzed, and the results demonstrated that ZRP exerted significant anti-diarrhea effect on rats with diarrhea due to spleen yang deficiency caused by folium sennae.

Brain-gut peptides are peptide hormones with special biological functions that are distributed in the gastrointestinal tract and central nervous system [15]. Recent studies have demonstrated that the expression of some brain-gut peptides is abnormal in a spleen-deficiency state, including expression of CCK, ghrelin, vasoactive intestinal peptide (VIP), and $\beta$-EP [15]. CCK functions in gallbladder contraction and stimulation of pancreatin secretion, enhances pancreatin activity, stimulates gastrointestinal muscles, and promotes peristalsis of the distal duodenum and jejunum [16]. Ghrelin is mainly produced by the stomach and acts on the central nervous system. It is widely distributed in the central nervous system and digestive system, and it can promote appetite [17]. Vasoactive intestinal peptide (VIP) is an inhibitory neurotransmitter that inhibits gastrointestinal motility [18]. The results of the present study suggest that ZRP exerts its antidiarrhea effect on rats by regulating levels of brain-gut peptides CCK, ghrelin, and VIP.

\section{CONCLUSION}

The present study is the first on polysaccharides from the rhizome of $Z$. officinale (ZRP) using RSM based on BBD. The results obtained indicate that RSM based on BBD is suitable for optimizing ZRP extraction, and subsequent experiments show that ZRP exerts significant anti-diarrhea effects in rats with diarrhea induced by folium sennae via regulation of the levels of brain-gut peptides.

\section{DECLARATIONS}

\section{Conflict of Interest}

No conflict of interest associated with this work.

\section{Contribution of Authors}

We declare that this work was done by the author(s) named in this article and all liabilities pertaining to claims relating to the content of this article will be borne by the authors. In addition, all authors read and approved the manuscript for publication. For preparation of the paper, ShuBin Li conceived and designed the study; DongMei Su, Jun-Biao Long and Xin-Ping Liu done the experiments; Dong-Mei $\mathrm{Su}$ and Jun-Biao Long analyzed the data; Dong-Mei Su and ShuBin Li wrote the manuscript.

\section{REFERENCES}

1. Li J, Du YW, Zhou DW, Gao CY. A comparative study of the compatibility of Wuweizi powder and Sishen pill on diarrhea of spleen deficiency effect. Shaanxi J Tradit Chin Med 2000; 28(7): 915-917.

2. Peng $W$, Shen $H$, Lin B, Han P, Li CH, Zhang QY, Ye BZ, Rahman K, Xin HL, Qin LP, Han T. Docking study and antiosteoporosis effects of a dibenzylbutane lignan isolated from Litsea cubeba targeting Cathepsin $K$ and MEK1. Med Chem Res 2018, 27:2062-2070. 
3. Zhou ZY. Medicine of traditional Chinese Medicine. Beijing: China Press of Traditional Chinese Medicine; 2002: 234-241 p.

4. Chinese Pharmacopoeia Commission. Pharmacopoeia of the People's Republic of China Part l; Beijing: People's Medical Publishing House; 2015. 14 p.

5. Sun FJ, Li ZL, Qian SH, Pu SB. Research advances on the Zingiberis rhizome. Chin Wild Plant Resour 2015; 34(3): 34-37.

6. World Medical Organization. Declaration of Helsinki. British Medical Journal (7 December) 1996; 313(7070): 1448-1449.

7. Dubois M, Gilles KA, Hamilton JK, Rebers PA, Smith F. Colorimetric method for determination of sugars and related substances. Anal Chem 1956; 28: 350-356.

8. Yang YQ, Guo WF, Li RL, Gao XL, Wang J, Lin CQ, Xu $S F$, Chen WW. Establishment of spleen yang deficiency rat model and observation of the therapeutic effect of Lizhong Decoction. Tradit Chin Drug Res Clin Pharmac 2009; 20(1): 83-86.

9. Yin $X$, You $Q$, Jiang $Z$, Zhou $X$. Optimization for ultrasonic-microwave synergistic extraction of polysaccharides from Cornus officinalis and characterization of polysaccharides. Int J Biol Macromol 2016; 83: 226-232.

10. Bo R, Ma X, Feng Y, Zhu Q, Huang Y, Liu Z, Liu C, Gao $Z$, Hu $Y$, Wang $D$. Optimization on conditions of Lycium barbarum polysaccharides liposome by RSM and its effects on the peritoneal macrophages function. Carbohydr Polym 2015; 117: 215-222.

11. Zhao ZY, Zhang Q, Li YF, Dong LL, Liu SL. Optimization of ultrasound extraction of Alisma orientalis polysaccharides by response surface methodology and their antioxidant activities. Carbohydr Polym 2015; 119: 101-109.

12. Li Q, Zhang HY, Wu TS, Zhang Z, Yang GL. Research progress on animal modeling methods and model evaluation of spleen-yang deficiency. World Sci and Technol/Modernization Tradit Chin Med and Mater Med 2015; 17(08): 1721-1728.

13. Guo XM. Pharmacodynamic study of wenyun mixture's therapy to spleen diarrhea of mice [dissertation]. Nanjing University of Chinese Medicine 2014. 17p

14. $X u Q$, Wang JH, Wang RJ, Liu $X Q$, ChenZX, Wu YL. Effect of Bu Zhong Yi Qi Decoction on gastrin receptor binding in rats with spleen deficiency and its protective mechanism against gastric mucosal injury. J Guangzhou Univ Tradit Chin Med 2003; (1): 51-56.

15. Liu WA, Liu M, He YM, LV Y, Guan C, Chang XR. Advances in the study of brain-gut peptide in functional gastrointestinal diseases. J Emerg Tradit Chin Med 2012; 21(06): 951-953.

16. Wang DS, Wang YJ, Zhang LD, Wang J, Li RF, Liu XD, Liu HH, Cai JY. Effects of eye on VIP content and gene expression in colon tissue of IBS rats. Chin J Basic Med Tradit Chin Med 2011; 17(03): 314-316.

17. Liu JD. Comparative study of cAMP/PKA signal pathway in brain-gut peptide and small intestine tissue of Spleen Qi deficiency syndrome and spleen yang deficiency syndrome rats [dissertation]. Liaoning University of Traditional Chinese Medicine 2016; $p 61$.

18. Zhang ZZ, Kuang WH, Zhang JL, Chen HJ, LV DY, Wu $Y L$. Effects of Tong Xie Yao Fang on intestinal function and the level of vasoactive intestinal peptide in hypothalamus and colon of rats with chronic stress. $J$ Anhui Tradit Chin Med Col 2010; 29(5): 53-55. 